\title{
Distinct clinical and immunological features of SARS- CoV-2-induced multisystem inflammatory syndrome in children
}

Pui Y. Lee, ${ }^{1}$ Megan Day-Lewis, ${ }^{1}$ Lauren A. Henderson, ${ }^{1}$ Kevin G. Friedman, ${ }^{2}$ Jeffrey Lo, ${ }^{1}$ Jordan E. Roberts, ${ }^{1}$ Mindy S. Lo, ${ }^{1}$ Craig D. Platt, ${ }^{1}$ Janet Chou, ${ }^{1}$ Kacie J. Hoyt, ${ }^{1}$ Annette L. Baker, ${ }^{2}$ Tina M. Banzon, ${ }^{1}$ Margaret H. Chang, ${ }^{1}$ Ezra Cohen, ${ }^{1,3}$ Sarah D. de Ferranti, ${ }^{2}$ Audrey Dionne, ${ }^{2}$ Saddiq Habiballah, ${ }^{1}$ Olha Halyabar, ${ }^{1}$ Jonathan S. Hausmann, ${ }^{1,4}$ Melissa M. Hazen, ${ }^{1}$ Erin Janssen, ${ }^{1}$ Esra Meidan, ${ }^{1}$ Ryan W. Nelson, ${ }^{1}$ Alan A. Nguyen, ${ }^{1}$ Robert P. Sundel, ${ }^{1}$ Fatma Dedeoglu, ${ }^{1}$ Peter A. Nigrovic, ${ }^{1,5}$ Jane W. Newburger, ${ }^{2}$ and Mary Beth F. Son ${ }^{1}$

'Division of Immunology and 2Department of Cardiology, Boston Children's Hospital, Harvard Medical School, Boston, Massachusetts, USA. ${ }^{3}$ Division of Pediatric Rheumatology, Department of Pediatrics, Boston Medical Center, Boston, Massachusetts, USA. Division of Rheumatology and Clinical Immunology, Beth Israel Deaconess Medical Center, Boston, Massachusetts, USA. ${ }^{5}$ Division of Rheumatology, Inflammation, and Immunity, Brigham and Women's Hospital, Boston, Massachusetts, USA.

BACKGROUND. Pediatric SARS-CoV-2 infection can be complicated by a dangerous hyperinflammatory condition termed multisystem inflammatory syndrome in children (MIS-C). The clinical and immunologic spectrum of MIS-C and its relationship to other inflammatory conditions of childhood have not been studied in detail.

METHODS. We retrospectively studied confirmed cases of MIS-C at our institution from March to June 2020. The clinical characteristics, laboratory studies, and treatment response were collected. Data were compared with historic cohorts of Kawasaki disease (KD) and macrophage activation syndrome (MAS).

RESULTS. Twenty-eight patients fulfilled the case definition of MIS-C. Median age at presentation was 9 years (range: 1 month to 17 years); $50 \%$ of patients had preexisting conditions. All patients had laboratory confirmation of SARS-CoV-2 infection. Seventeen patients (61\%) required intensive care, including 7 patients $(25 \%)$ who required inotrope support. Seven patients (25\%) met criteria for complete or incomplete KD, and coronary abnormalities were found in 6 cases. Lymphopenia, thrombocytopenia, and elevation in inflammatory markers, D-dimer, B-type natriuretic peptide, IL-6, and IL-10 levels were common but not ubiquitous. Cytopenias distinguished MIS-C from KD and the degree of hyperferritinemia and pattern of cytokine production differed between MIS-C and MAS. Immunomodulatory therapy given to patients with MIS-C included intravenous immune globulin (IVIC) (71\%), corticosteroids (61\%), and anakinra (18\%). Clinical and laboratory improvement were observed in all cases, including 6 cases that did not require immunomodulatory therapy. No mortality was recorded in this cohort.

CONCLUSION. MIS-C encompasses a broad phenotypic spectrum with clinical and laboratory features distinct from KD and MAS.

FUNDING. This work was supported by the National Institutes of Health, National Institute of Arthritis and Musculoskeletal and Skin Diseases; the National Institute of Allergy and Infectious Diseases; Rheumatology Research Foundation Investigator Awards and Medical Education Award; Boston Children's Hospital Faculty Career Development Awards; the McCance Family Foundation; and the Samara Jan Turkel Center.

\section{Introduction}

Since the onset of the coronavirus disease 2019 (COVID-19) pandemic, millions of individuals have been infected with

\section{Related Commentary: 5681}

Authorship note: PYL, MDL, and LAH are co-first authors. Conflict of interest: The authors have declared that no conflict of interest exists. Copyright: () 2020, American Society for Clinical Investigation.

Submitted: June 8, 2020; Accepted: July 22, 2020; Published: October 5, 2020 Reference information: J Clin Invest. 2020;130(11):5942-5950.

https://doi.org/10.1172/JCl141113. severe acute respiratory syndrome coronavirus 2 (SARS-CoV-2) and more than 500,000 deaths have been recorded worldwide. SARS-CoV-2 affects every age group, although mortality correlates most strongly with advanced age as well as the presence of preexisting medical conditions (1, 2). Early studies from China demonstrated a generally mild course in children with COVID-19 $(3,4)$, but recent reports from Europe and North America described a number of seriously ill children (5-10). In addition to cases that exhibit classic features of Kawasaki disease (KD), heterogeneous manifestations of systemic inflammation and shock are increasingly reported $(11,12)$. These cases have been categorized as pediatric multisystem inflammatory syndrome tempo- 


\section{Table 1. Demographic and clinical characteristics of patients with MIS-C}

\begin{tabular}{|c|c|}
\hline Number of patients ${ }^{A}$ & 28 \\
\hline CDC case definition & $100 \%$ \\
\hline WHO case definition & $93 \%$ \\
\hline \multicolumn{2}{|l|}{ Demographics } \\
\hline Age, median (range) & 9.0 y $(0.1-17)$ \\
\hline Female & $43 \%$ \\
\hline White & $36 \%$ \\
\hline Black & $18 \%$ \\
\hline Hispanic & $43 \%$ \\
\hline Preexisting condition & $50 \%$ \\
\hline Duration of symptoms at admission & $5 d(1-10)$ \\
\hline Reported contact with COVID-19 & $29 \%$ \\
\hline SARS-CoV-2 testing & $100 \%$ \\
\hline SARS-CoV-2 serology positive & $95 \%(18 / 19)$ \\
\hline Nasopharyngeal swab PCR positive & $61 \%(17 / 28)$ \\
\hline \multicolumn{2}{|l|}{ Details of hospitalization } \\
\hline Intensive care unit & $61 \%$ \\
\hline Inotropes & $25 \%$ \\
\hline Supplemental oxygen & $43 \%$ \\
\hline Noninvasive ventilation & $25 \%$ \\
\hline Invasive ventilation ${ }^{B}$ & $4 \%$ \\
\hline \multicolumn{2}{|l|}{ Clinical manifestations } \\
\hline Fever & $100 \%$ \\
\hline Conjunctivitis & $57 \%$ \\
\hline Gastrointestinal symptoms & $54 \%$ \\
\hline Hypotension / shock & $54 \%$ \\
\hline Skin rash & $36 \%$ \\
\hline Mucositis & $25 \%$ \\
\hline Extremity swelling / erythema & $21 \%$ \\
\hline Acute kidney injury & $21 \%$ \\
\hline \multicolumn{2}{|c|}{$\begin{array}{l}{ }^{A} \text { All } 28 \text { patients were included in percentage calculations unless noted } \\
\text { otherwise. Bntubation was not required for any patient. One patient } \\
\text { with tracheostomy at baseline required mechanical ventilation during } \\
\text { acute illness. }\end{array}$} \\
\hline
\end{tabular}

rally associated with COVID-19 (PMIS) by the Royal College of Pediatrics and Child Health (13) and as multisystem inflammatory syndrome in children (MIS-C) by the World Health Organization (WHO) and the Centers for Disease Control and Prevention (CDC) $(14,15)$. The risk factors that predispose some children to develop this inflammatory response to SARS-CoV-2 infections are not fully understood. As in adults with COVID-19, a disproportionate number of patients with MIS-C belong to minority racial and/or ethnic groups (5, 8-10).

Identification of MIS-C is important because severe organ dysfunction and death have been reported in young patients $(8,9$, 11). Timely initiation of immunomodulatory agents may help correct the hyperinflammatory state and prevent end-organ damage. However, our understanding of the clinical spectrum of MIS-C remains limited. Excessive production of inflammatory cytokines, as a direct reaction to the virus or as a postinfectious phenomenon, is linked to disease severity in adults (16). Corticosteroids and biologics that target the cytokines IL- 1 and IL- 6 are increasingly used to treat the cytokine storm associated with COVID-19 (17-19).
Whether MIS-C reflects a parallel COVID-19-associated hyperinflammatory state in the pediatric population is an important question to investigate. Moreover, how the clinical and laboratory features of MIS-C relate to known syndromes of hyperinflammation and cytokine storm in children, including KD and macrophage activation syndrome (MAS), has not been studied in detail. In this study, we describe 28 confirmed cases of MIS-C at Boston Children's Hospital and discuss the immunologic findings and management of these patients. We compare the characteristics of this novel syndrome with historic cohorts of patients with KD and MAS.

\section{Results}

Clinical manifestations and diagnostic findings of MIS-C. Demographic and clinical manifestations of our cohort of patients with MIS-C are summarized in Table 1 . The median age was 9 years (range: 1 month-17 years) and 57\% were male. In this cohort, 18\% of patients were Black and $43 \%$ were Hispanic, which contrasts with the state demographics (9\% Black and 12\% Hispanic) (20). Half $(n=14)$ of these patients had preexisting medical conditions, including obesity $(n=4)$, asthma $(n=3)$, congenital heart disease $(n=1)$, sickle cell anemia $(n=1)$, mitochondrial disorder $(n=1)$, autism $(n=1)$, and chromosomal abnormalities $(n=1)$. Interestingly, 2 patients had a history of KD, 1 of whom had developed a coronary aneurysm with an earlier episode. The median duration of symptoms prior to hospitalization was 5 days. All cases had confirmatory evidence of SARS-CoV-2 infection. PCR was performed from nasopharyngeal swab sampling for all patients and 17 of 28 (61\%) were positive. Nineteen patients were tested for antibodies and 18 (95\%) possessed antibodies to the virus.

Highlighting the severity of MIS-C, 17 patients (61\%) required admission to the intensive care unit (ICU). Hypotension and/or shock were noted in 15 of 28 patients (54\%) and inotropes were required in 7 cases (25\%). Common features of MIS-C included gastrointestinal symptoms (54\%), conjunctivitis (57\%), and skin rash (36\%) (Table 1). Unlike adults hospitalized due to COVID-19, less than half of our patients experienced respiratory impairment (defined by the requirement for supplemental oxygen or additional respiratory support). Noninvasive ventilation (bilevel positive airway pressure or continuous positive airway pressure) was provided to 7 patients (25\%) while 4 other patients (14\%) briefly required supplemental oxygen by nasal cannula; no patient required intubation or extracorporeal membrane oxygenation. Evidence of acute kidney injury was found in 6 patients (21\%).

Echocardiogram identified left ventricular dysfunction (defined by ejection fraction $<55 \%$ ) in 11 patients (39\%), including 2 with an ejection fraction less than $30 \%$ (Table 2). The prevalence of cardiac stress was further supported by elevated serum B-type natriuretic peptide (BNP) in approximately half of cases. New coronary artery abnormalities were found in 6 cases (further discussed below). Chest x-ray noted focal consolidations or opacities in 10 of 26 patients (38\%). Chest computerized tomography was obtained in 3 patients with respiratory insufficiency and demonstrated ground glass opacities $(n=2)$ and multifocal opacities $(n$ $=1$ ). Abdominal ultrasound was performed for 6 patients and the results were notable for mesenteric adenitis $(n=2)$, trace ascites $(n$ $=1$ ), and increased liver or kidney echogenicity ( $n=1$ each). 


\section{Table 2. Radiographic and laboratory findings in patients with MIS-C}

\begin{tabular}{|c|c|}
\hline \multicolumn{2}{|l|}{ Echocardiography } \\
\hline Ejection fraction <55\% & $39 \%(11 / 28)$ \\
\hline Dilated coronary vesse ${ }^{A}$ & $7 \%(2 / 28)$ \\
\hline Coronary aneurysm ${ }^{\mathrm{B}}$ & $14 \%(4 / 28)$ \\
\hline \multicolumn{2}{|l|}{ Chest x-ray } \\
\hline Focal consolidation / opacity & $14 \%(4 / 28)$ \\
\hline Prominent vasculature & $15 \%(4 / 26)$ \\
\hline Enlarged cardiac silhouette & $8 \%(3 / 26)$ \\
\hline Pleural effusion & $12 \%(2 / 26)$ \\
\hline Peribronchial thickening & $12 \%(2 / 26)$ \\
\hline \multicolumn{2}{|l|}{ Cardiac biomarkers } \\
\hline $\mathrm{BNP}>100 \mathrm{pg} / \mathrm{mL}$ & $52 \%(12 / 23)$ \\
\hline Troponin $>0.09 \mathrm{ng} / \mathrm{mL}$ & $27 \%(6 / 22)$ \\
\hline \multicolumn{2}{|l|}{ Inflammatory markers } \\
\hline Procalcitonin $\geq 0.1 \mathrm{ng} / \mathrm{mL}$ & $96 \%(24 / 25)$ \\
\hline $\mathrm{CRP}>0.5 \mathrm{mg} / \mathrm{dL}$ & $93 \%(26 / 28)$ \\
\hline Ferritin $>200 \mathrm{ng} / \mathrm{mL}$ & $86 \%(24 / 28)$ \\
\hline $\mathrm{ESR}>30 \mathrm{~mm} / \mathrm{h}$ & $63 \%(15 / 24)$ \\
\hline \multicolumn{2}{|l|}{ Hematologic parameters } \\
\hline $\mathrm{ALC}<2 \times 103 / \mathrm{mL}$ & $75 \%(21 / 28)$ \\
\hline Platelets $<200 \times 103 / \mathrm{mL}$ & $64 \%(18 / 28)$ \\
\hline $\mathrm{WBC}>10 \times 103 / \mathrm{mL}$ & $39 \%(11 / 28)$ \\
\hline Hemoglobin < $11 \mathrm{~g} / \mathrm{dL}$ & $32 \%(9 / 28)$ \\
\hline $\mathrm{WBC}<5 \times 103 / \mathrm{mL}$ & $25 \%(7 / 28)$ \\
\hline $\mathrm{ANC}<2 \times 103 / \mathrm{mL}$ & $18 \%(5 / 28)$ \\
\hline \multicolumn{2}{|l|}{ Coagulation parameters } \\
\hline D-dimer $>0.5 \mathrm{mg} / \mathrm{mL}$ & $96 \%(26 / 27)$ \\
\hline Fibrinogen $>400 \mathrm{mg} / \mathrm{dL}$ & $71 \%(15 / 21)$ \\
\hline PT $>14.6$ seconds & $62 \%(16 / 26)$ \\
\hline PTT > 37 seconds & $38 \%(10 / 26)$ \\
\hline \multicolumn{2}{|l|}{ Organ function parameters } \\
\hline $\mathrm{LDH}>250 \mathrm{U} / \mathrm{L}$ & $74 \%(14 / 19)$ \\
\hline AST $>50 \mathrm{U} / \mathrm{L}$ & $46 \%(13 / 28)$ \\
\hline $\mathrm{ALT}>40 \mathrm{U} / \mathrm{L}$ & $32 \%(9 / 28)$ \\
\hline BUN > $18 \mathrm{mg} / \mathrm{dL}$ & $25 \%(7 / 28)$ \\
\hline Creatinine $>0.8 \mathrm{mg} / \mathrm{dL}$ & $21 \%(6 / 28)$ \\
\hline \multicolumn{2}{|c|}{$\begin{array}{l}{ }^{A} Z \text { score of the left anterior descending and/or right coronary artery is } \\
\text { between } 2.0 \text { and }<2.5 .{ }^{B} Z \text { score of the left anterior descending, left } \\
\text { main coronary artery and/or right coronary artery is } \geq 2.5 \text {. One patient } \\
\text { with known small coronary aneurysm from a previous episode of } K D \\
\text { was not counted. }\end{array}$} \\
\hline
\end{tabular}

Laboratory features of our patients with MIS-C included elevated inflammatory markers, lymphopenia, and thrombocytopenia (Table 2). Eighteen patients $(64 \%)$ had low platelet count $(<$ $200 \times 10^{3} / \mu \mathrm{L} ; 5$ patients $\left.<100 \times 10^{3} / \mu \mathrm{L}\right)$ while 21 patients $(75 \%)$ had low absolute lymphocyte count $\left(<2 \times 10^{3} / \mu \mathrm{L}\right)$. It is notable that no single inflammatory marker (C-reactive protein, erythrocyte sedimentation rate, procalcitonin, or ferritin) was elevated in all cases, but each patient demonstrated elevation of at least one of these markers. The majority of patients displayed evidence of coagulopathy highlighted by elevated D-dimers (96\%) and prolonged prothrombin time (62\%). Features associated with ICU admission included hypotension, high procalcitonin levels, and elevated BNP levels (Supplemental Table 2; supplemental material available online with this article; https://oi.org/10.1172/ JCI141113DS1). BNP levels correlated inversely with ejection fraction (Supplemental Figure 1). Taken together, these data illustrate the broad spectrum of clinical manifestations and disease severity in patients hospitalized with MIS-C.

Comparison of MIS-C and KD. To understand the overlap between KD and MIS-C, we assessed the clinical and laboratory features of $\mathrm{KD}$ in patients with MIS-C and compared them with the 40 consecutive cases of KD at our center between May 2019 and February 2020. Patients with MIS-C exhibited a broader age range compared with the early childhood predominance seen in KD (Figure 1A; median 9 vs. 2 years; $P<0.0001$ ). The prevalence of each KD feature was numerically lower in MIS-C than in KD (Figure 1B). Two patients with MIS-C had 4 clinical features consistent with a diagnosis of complete KD (Figure 1C). Among 13 patients with 2 or 3 features of KD, 5 met the supplemental criteria for consideration of incomplete KD. Eleven patients (39\%) had 1 or fewer criterion of KD by history and physical exam (Figure 1C). Thus, 7 of 28 patients with MIS-C (25\%) met criteria for complete or incomplete $\mathrm{KD}$. It is worth noting that during the study period between March 17, 2020, and June 6, 2020, we also diagnosed 8 cases of KD that did not satisfy the definitions for MIS-C, an incidence of KD comparable to what has been observed at our institution in previous years (Supplemental Figure 2A).

In the absence of a diagnostic test for $\mathrm{KD}$, the development of coronary artery aneurysms confirms the diagnosis in patients who meet criteria for KD (21). New coronary artery abnormalities (defined as $Z$ score $>2.0$ ) were found in 6 patients with MIS-C (21\%) compared with 14 of 40 patients (35\%) in the KD group ( $P$ $=0.12$, Fisher's exact test; Figure 1D). Details of these cases are provided in Supplemental Table 3. One patient with a preexisting small aneurysm from a prior episode of $\mathrm{KD}$ was not counted as a new event; the size of the aneurysm was unchanged from baseline and did not progress on serial echocardiography during the course of MIS-C. Two patients, including another with prior history of KD but no prior aneurysms, had dilation of coronary vessels ( $Z$ score between $>2.0$ and $\leq 2.5$ of the right coronary artery [RCA]). Small aneurysms as defined by a $Z$ score 2.5 to $\leq 5.0$ (21) were found in 4 patients $(Z$ score $=2.6$ of the left anterior descending coronary artery, 3.0 of the left main coronary artery, 3.0 of the RCA, and 3.6 of the RCA) who met 2 or fewer KD criteria, including one who did not meet any KD criterion. Whereas coronary aneurysms were present only in a few patients, ventricular dysfunction was common in MIS-C. Patients with MIS-C displayed significantly lower ejection fractions compared with the KD group (Figure 1E).

Multiple laboratory features distinguished MIS-C from $\mathrm{KD}$, including lower white blood cell count, lymphocyte count, platelet count, and erythrocyte sedimentation rate (ESR) (Figure $1 \mathrm{~F}$ ). Anemia was more common in KD. CRP levels were comparable whereas transaminase elevation was more prominent in MIS-C. Since BNP, ferritin, and D-dimer levels were not consistently obtained in our historic cohort of patients with KD, we were unable to comment on the differences in these parameters between children with KD and those with MIS-C. Thus, the clinical spectrum of MIS-C overlaps with KD but also exhibits important differences; notably, ventricular dysfunction is more 
A

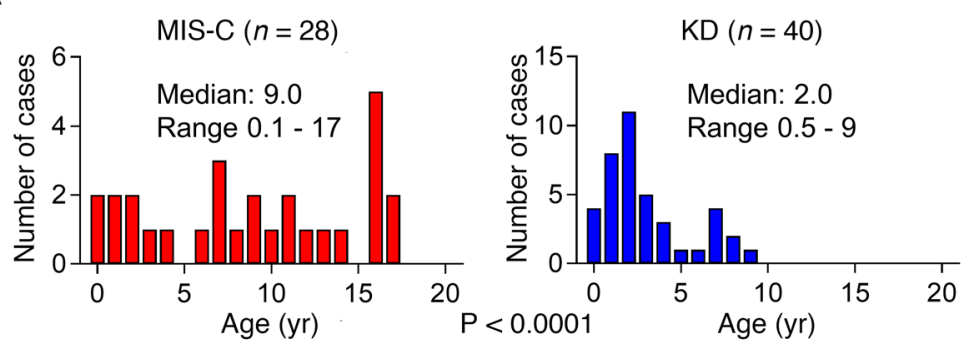

C

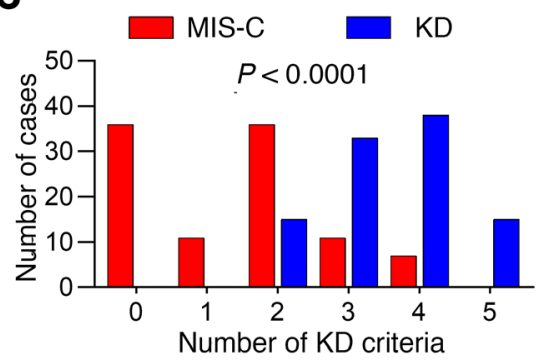

D

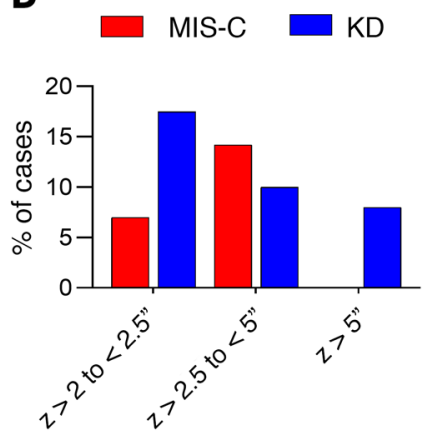

B

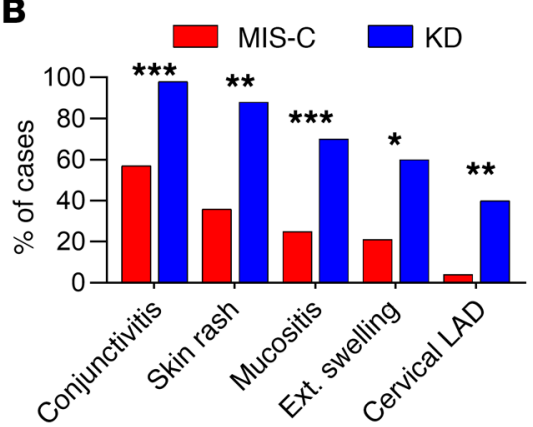

E
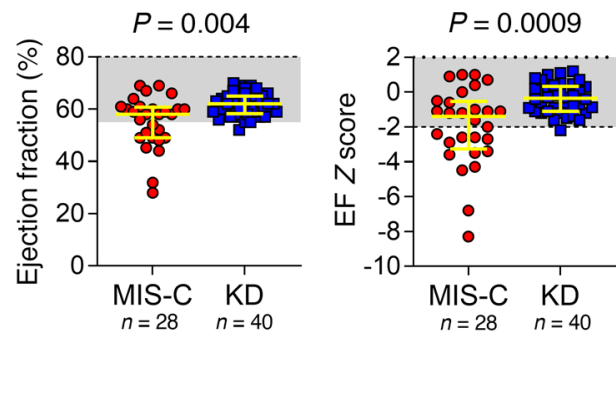

F
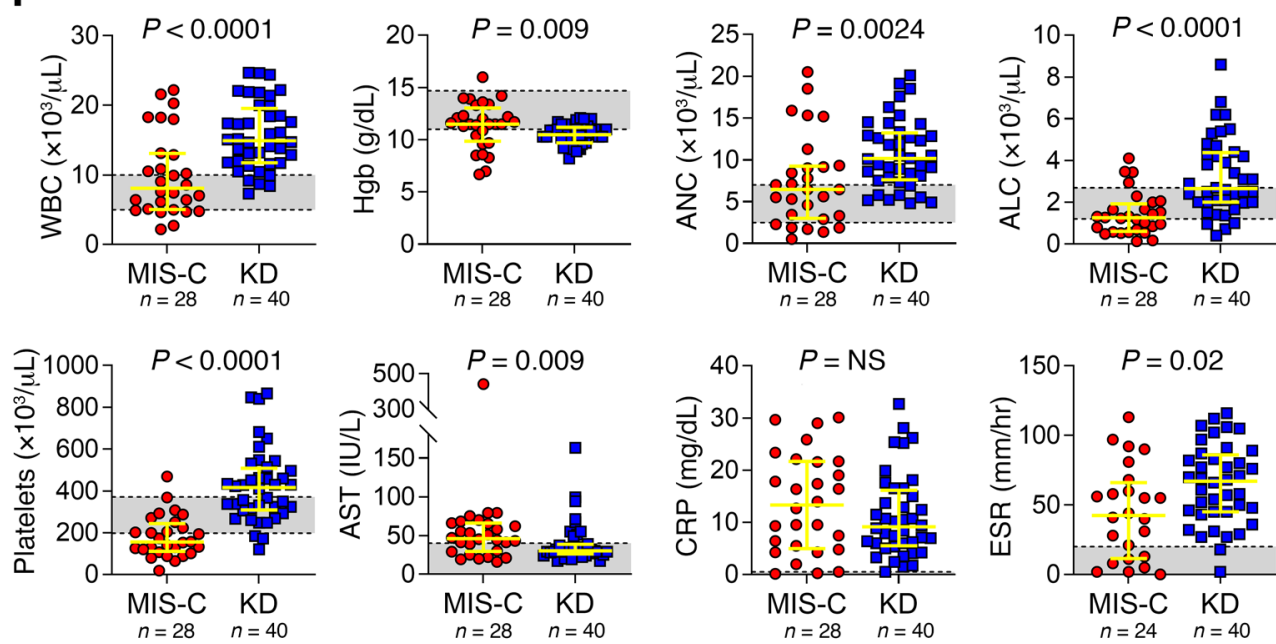

Figure 1. Comparison of clinical features and laboratory parameters in patients with MIS-C versus those with KD. MIS-C $n=28 ; \mathrm{KD} n=40$. (A) Histogram display of age range of MIS-C and KD patients. Comparisons of (B) prevalence of individual KD features $\left({ }^{*} P<0.01 ;{ }^{* *} P<0.001\right.$; ${ }^{* * *} P<0.0001$, Fisher's exact test), (C) the number of KD diagnostic feature $\left(P<0.0001, \chi^{2}\right.$ test), (D) prevalence of coronary abnormalities $\left(P>0.05, \chi^{2}\right.$ test), and (E) ejection fraction as an index of left ventricular function (by percentage and by $Z$ score) in the MIS-C and KD groups. (F) Comparison of key laboratory parameters including white blood cell count (WBC), hemoglobin, absolute neutrophil count (ANC), absolute lymphocyte count (ALC), platelet count, aspartate transaminase (AST), erythrocyte sedimentation rate (ESR), and C-reactive protein (CRP). Median with interquartile range and $P$ value by Mann-Whitney $U$ test are displayed for each plot. Gray shading indicates the normal range for laboratory parameters.

common in MIS-C, and coronary artery aneurysms can develop in patients with few to no KD features.

Immunologic features of MIS-C and comparison with MAS. Studies of COVID-19 in adults have noted that lymphopenia and excess cytokine production are associated with disease severity (16). In our MIS-C cohort, the majority of patients exhibited reduced numbers of $\mathrm{CD} 4^{+}$and $\mathrm{CD} 8^{+} \mathrm{T}$ lymphocytes and NK cells, whereas B lymphocytes were reduced less consistently (Figure 2A). Baseline total antibody levels were largely preserved, although mild hypogammaglobulinemia was found in several patients (Figure
2B). Serum cytokine analysis showed elevated IL-6, IL-10, and sIL2R levels as common features in MIS-C (Figure 2, C and D).

The constellation of cytopenias, coagulopathy, hyperferritinemia, and elevated sIL2R levels is reminiscent of MAS, a cytokine storm typically associated with systemic juvenile idiopathic arthritis (JIA), infection, and malignancy. Compared with a historic cohort of patients with MAS (associated with systemic JIA-associated or infection; see Methods), the increase in sIL2R was less striking in MIS-C (median $2011 \mathrm{pg} / \mathrm{mL}$ vs. $5099 \mathrm{pg} / \mathrm{mL}$ in MAS group; $P=0.02$, Figure 2D). Ferritin levels were significantly higher 
A
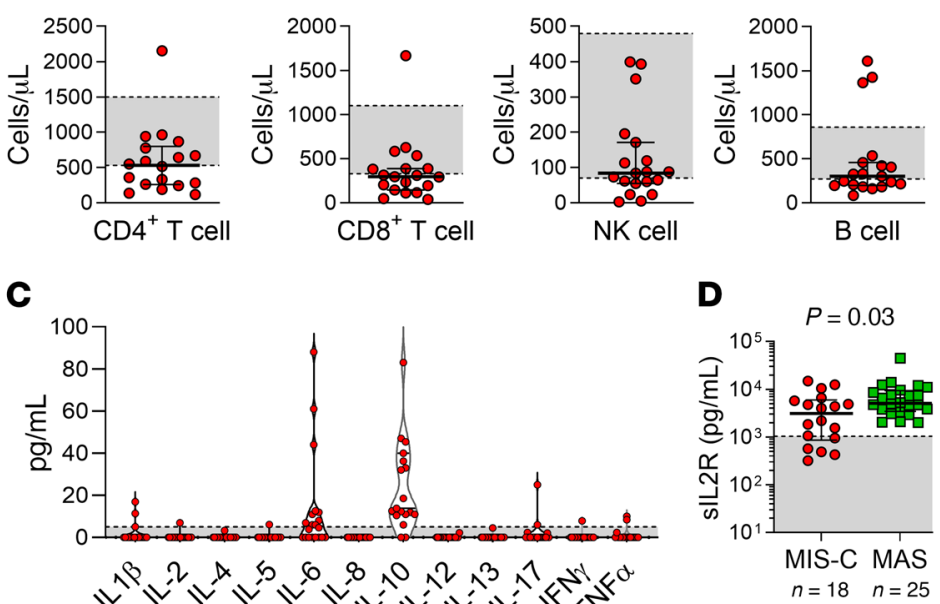

B
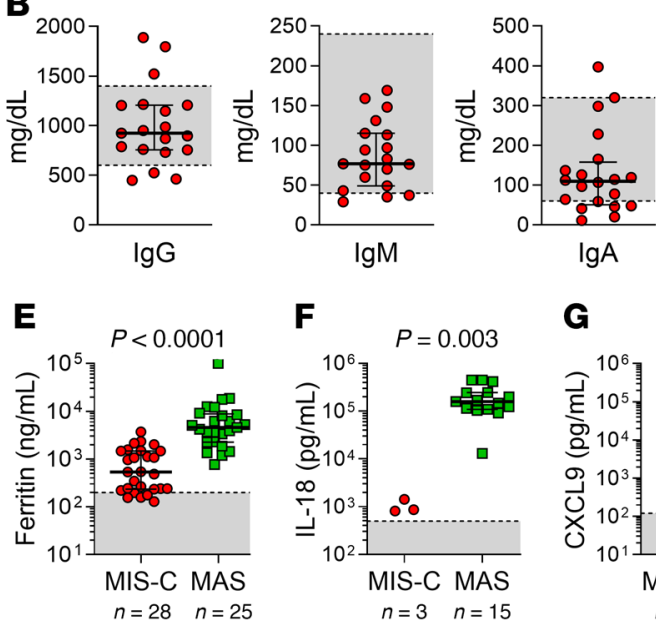

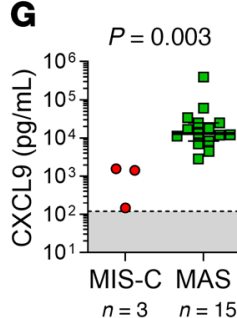

Figure 2. Immunologic profile of patients with MIS-C and comparison with MAS. (A) Quantitation of lymphocyte populations, (B) baseline immunoglobulin levels, (C) serum cytokine levels in MIS-C patients ( $n=15-22$ for each panel). (D-C) Comparison of (D) soluble IL2 receptor levels, (E) ferritin, (F) IL-18, and (C) CXCL9 levels in MIS-C with a cohort of patients with MAS associated with systemic JIA or infection. Median with interquartile range and $P$ value by Mann-Whitney $U$ are displayed for each plot. Gray shading indicates the normal range for laboratory parameters.

in MAS group compared with MIS-C (median $4594 \mathrm{ng} / \mathrm{mL}$ vs. $537 \mathrm{ng} / \mathrm{mL} ; P<0.0001$; Figure 2E). Unlike the log-fold increases typical for MAS (Figure 2, F and G), IL-18 and CXCL9 (a surrogate marker for interferon- $\gamma$ ) were only mildly elevated in several MIS-C patients (all of whom required ICU admission and had ferritin $>1500 \mathrm{ng} / \mathrm{mL}$ ), Overall, the immunologic features of MIS-C did not appear to closely resemble MAS.

Treatment and outcome. Currently, there is no standardized treatment for MIS-C. Immunomodulatory medications were administered to 22 of the 28 patients on average within 1.5 days of admission. Most patients (71\%) received IVIG at a dose of 2 $\mathrm{g} / \mathrm{kg}$ and $61 \%$ were given methylprednisolone at doses ranging from 1 to $4 \mathrm{mg} / \mathrm{kg} /$ day (Table 3). Anakinra (recombinant IL-1 receptor antagonist) was used in 5 cases (18\%) at doses ranging from 5 to $13 \mathrm{mg} / \mathrm{kg} /$ day, typically in patients refractory to IVIG and glucocorticoids. Anakinra monotherapy was used in one child with sickle cell anemia to avoid glucocorticoids, which are known to complicate vasoocclusive pain crises (22). Clinical improvement was seen in all cases, with resolution of fever, cessation of inotrope treatment, and improvement of inflammatory markers. In those treated with immunomodulatory medications, fever resolved after 4.0 days (median) of admission and CRP invariably decreased (mean time to a 50\% CRP reduction: 5.3 days). Ferritin and D-dimer did not respond as quickly to treatment as CRP and often remained stably elevated for several days before consistent declines were observed. The mean time to $50 \%$ reduction in ferritin and D-dimer levels were 13.4 days and 7.2 days, respectively.

The 6 cases with new coronary artery abnormalities were treated with IVIG (4 also received corticosteroids) and 3 showed normalization of coronary vessel size $(Z$ score $<2)$ after treatment. Another patient showed improvement of RCA dilation (from $Z$ score 3.1 to 2.2$)$ and 2 patients had stable coronary aneurysm $(Z$ score $\leq 3.0$ ) without further progression after treatment on follow-up echocardiogram studies. Interestingly, 6 patients in the cohort showed spontaneous improvement of fever and inflammatory markers without immunomodulatory therapy. Two of these patients were afebrile after admission and the median time to fever resolution in the other 4 patients was 2.5 days. Coronary artery dimensions were normal throughout the course of these 6 patients who were not treated.

Remdesivir was given on a compassionate-use basis in 7 patients and hydroxychloroquine was used in 1 patient. More than half of the cohort received at least one antibiotic. Due to the risk of thrombosis associated with COVID-19 (23) and evidence of coagulopathy in MIS-C, most patients also received anticoagulation therapy. Six patients (21\%) received aspirin alone, 5 (18\%) received enoxaparin alone and 13 patients received both (46\%). Low-dose aspirin (3-6 mg/kg/day was used most frequently ( $n$ = 14) while moderate dosing $(20-50 \mathrm{mg} / \mathrm{kg} /$ day $)$ was given to 5 patients. No thrombotic event was detected in our patients during their hospital course.

With rapid diagnosis and treatment initiation, clinical outcomes in our cohort of patients with MIS-C were generally favorable. The median duration of hospitalization was 8.0 days $(9.5$ days for patients who required ICU stay and 4.0 days for patients who did not receive ICU care). Patients treated with glucocorticoids were discharged from the hospital with a planned steroid taper that typically lasted for 2 to 4 weeks, as guided by continued improvement of symptoms and serologic markers of inflammation. All patients were discharged home and none had died.

\section{Discussion}

In the early phases of the COVID-19 pandemic, children were thought to be largely spared from much of the morbidity and mortality related to SARS-CoV-2 infection $(3,4)$. However, recent case series from Europe and the United States have raised concerns about a hyperinflammatory process associated with COVID-19 in children, now collectively defined as MIS-C $(5,6,8-10,12,24,25)$. In addition to persistent fever and nonspecific features of viral ill- 
Table 3. Treatment approaches and outcome in patients with MIS-C

\begin{tabular}{|c|c|c|c|}
\hline & $\begin{array}{c}\text { Total } \\
n=28\end{array}$ & $\begin{array}{c}\text { ICU } \\
n=17\end{array}$ & $\begin{array}{c}\text { Non-ICU } \\
n=11\end{array}$ \\
\hline Duration of hospitalization, days, median & 8.0 & 9.5 & 4.0 \\
\hline Discharge from hospital & $100 \%$ & - & - \\
\hline Death & $0 \%$ & - & - \\
\hline \multicolumn{4}{|l|}{ Immunomodulatory therapy, $n(\%)^{A}$} \\
\hline None & $6(21 \%)$ & $3(18 \%)$ & $3(27 \%)$ \\
\hline IVIG only & $4(14 \%)$ & $0(0 \%)$ & $4(36 \%)$ \\
\hline Methylprednisolone only & $1(4 \%)$ & $1(6 \%)$ & $0(0 \%)$ \\
\hline Anakinra only & $1(4 \%)$ & $0(0 \%)$ & $1(9 \%)$ \\
\hline IVIG + methylprednisolone & $12(43 \%)$ & $9(53 \%)$ & $3(27 \%)$ \\
\hline IVIG + methylprednisolone + anakinra & $4(14 \%)$ & $4(24 \%)$ & $0(0 \%)$ \\
\hline \multicolumn{4}{|l|}{ Antimicrobial therapy, $n$ (\%) } \\
\hline Remdesivir & $7(25 \%)$ & $6(35 \%)$ & $1(9 \%)$ \\
\hline Antibiotics & $15(54 \%)$ & $12(71 \%)$ & $3(27 \%)$ \\
\hline \multicolumn{4}{|l|}{ Anticoagulation therapy, $n(\%)^{\mathrm{B}}$} \\
\hline None & $4(14 \%)$ & $2(12 \%)$ & $2(18 \%)$ \\
\hline Aspirin & $19(68 \%)$ & $13(76 \%)$ & $6(55 \%)$ \\
\hline Enoxaparin & $18(64 \%)$ & $13(76 \%)$ & $5(45 \%)$ \\
\hline \multicolumn{4}{|c|}{$\begin{array}{l}{ }^{A} \mathrm{~A} \text { total of } 20 \text { patients were treated with IVIG ( } 2 \mathrm{gm} / \mathrm{kg}, n=13 ; 1 \mathrm{gm} / \mathrm{kg}, n=6 ; 0.5 \mathrm{gm} / \\
\mathrm{kg}, n=1) \text {. A second dose of IVIG was given in } 2 \text { cases. A total of } 17 \text { patients were treated } \\
\text { with methylprednisolone ( } \leq 2 \mathrm{mg} / \mathrm{kg} / \mathrm{day}, n=13 ; 3-4 \mathrm{mg} / \mathrm{kg} / \mathrm{day}, n=4) \text {. A total of } 5 \\
\text { patients were treated with anakinra at doses ranging from } 5-13 \mathrm{mg} / \mathrm{kg} / \mathrm{day} .{ }^{\mathrm{B}} \mathrm{A} \text { total of } \\
19 \text { patients were treated with aspirin (ASA) ( } 3-6 \mathrm{mg} / \mathrm{kg} / \mathrm{day}, n=14 ; 20-50 \mathrm{mg} / \mathrm{kg} / \mathrm{day} \text {, } \\
n=5 \text { ). A total of } 18 \text { patients were treated with enoxaparin (prophylactic dosing, } n=13 \text {; } \\
\text { treatment dosing, } n=5 \text { ). }\end{array}$} \\
\hline
\end{tabular}

prominent KD features developed cardiac complications, demonstrating the importance of cardiac evaluation for all patients with MIS-C. Based on prior reports as well as our findings, a KD-like illness appears to be part of the clinical spectrum of MIS-C, but the spectrum further encompasses findings that are uncommon in KD. Remarkably, 2 of 28 patients with MIS-C had previously experienced KD, suggesting potential shared genetic susceptibilities between the 2 conditions. On the other hand, the surprising absence of reported MIS-C-like illness in Asia, where $\mathrm{KD}$ is most prevalent, suggests that MIS-C and KD likely have distinct genetic predispositions. Genomic studies will be required to resolve the contribution of host genetics to the development of MIS-C. Geographic differences, variabilities in population genetics, and divergence of SARS-CoV-2 strains may further contribute to the pleiotropic hyperinflammatory phenotypes associated with COVID-19.

Some of the laboratory findings in MIS-C are suggestive of a MAS-like cytokine storm. However, differences in the degree of elevation of markers such as ferritin, IL-18, and CXCL9 suggest that the pathogenic cytokines driving MIS-C likely diverge from those driving MAS. Interestingly, the immunologic findings in MIS-C were similar to the profile described in severe cases of COVID-19 in adults. Profound lymphopenia and elevated levels of IL-6, IL-10, and sIL2R were all associated with disease ness, these children may exhibit heart failure, shock, and coronary artery abnormalities.

Our initial experience with 28 cases at Boston Children's Hospital showed that MIS-C spans a spectrum of clinical features with notable variability in age and severity. In comparison to reports focused on patients in the ICU $(5,12)$, our study captured a greater spectrum of MIS-C manifestations and immunologic findings by including all hospitalized patients who fulfill the case definition. We observed a striking overrepresentation of Black and Hispanic racial and ethnic groups, reflecting the disproportionate burden of illness that these minority groups are experiencing during the pandemic $(26,27)$. Preexisting conditions including obesity and asthma are shared risk factors for MIS-C and COVID-19 in adults (28). Unlike adults with severe COVID-19, acute respiratory distress syndrome (ARDS) was not a feature in these children. Instead, MIS-C is associated with a preponderance of cardiac complications, including ventricular dysfunction and coronary abnormalities. The degree of inflammation as measured by CRP and procalcitonin is much greater in MIS-C compared with children with COVID-19 pneumonia $(3,4)$. Clinical vigilance and early institution of multidisciplinary care were associated with a good prognosis in our patients, although it is noteworthy that 6 patients in our cohort improved with supportive care alone, possibly indicating that immunomodulatory treatment may not be required for milder cases.

While we noted features of KD in some cases, the severity of illness and ventricular dysfunction are uncharacteristic for $\mathrm{KD}$ and conversely, rash and conjunctivitis are common in viral syndromes and not specific for KD. Importantly, patients without severity in the adult COVID-19 population (16). However, IL-6 levels in our MIS-C patient were generally lower while IL-10 and sIL2R levels were higher compared with severe COVID-19 in adults $(16,29,30)$. Due to potential variations between assays, direct comparison of adult and pediatric samples is needed to confirm these observations. While we did not measure serum IL- 6 in our KD and MAS cohorts, elevated IL- 6 levels have also been linked to these conditions $(31,32)$.

Massachusetts has among the most confirmed COVID-19 cases in the United States, with more than 100,000 cases reported (20). Community spread of the virus in the state was first noted in early March 2020 and peaked by late April 2020 for adults of all age groups (Supplemental Figure 2B). Approximately $5 \%$ of confirmed cases were children under 19 years of age and the trajectory of pediatric cases lagged behind all adult age groups. Similar to reports from Europe, the influx of MIS-C cases at our institution occurred 3 to 4 weeks after the peak of adult COVID-19 (Supplemental Figure 2C). The earliest cases of MIS-C in our institution can be traced to as early as March 17, 2020.

It has been hypothesized that MIS- $\mathrm{C}$ is an immune-mediated postinfectious process to SARS-CoV-2. While more than $60 \%$ of cases had detectable viral nucleic acids from nasopharyngeal aspirates, the presence of antibodies to SARS-CoV-2 in the majority of patients supports an interval of at least 1 to 2 weeks between initial infection and the onset of MIS-C. It is possible that nasopharyngeal aspirates reflect viral fragments that persist past active infection. Studies aimed to culture live SARS-CoV-2 virus from patients with MIS-C and gain a better understanding 
of the kinetics of antibody development to the virus are needed to better address this question.

The outcome for MIS-C was generally favorable in our cohort, with cardiopulmonary support and aggressive management of systemic inflammation. No mortality was observed in these 28 patients, whereas deaths have been reported in prior case series $(5,8,9,25)$. Our general experience with MIS-C was similar to the observations from the latest cohort studies $(12,24,25,33,34)$. The frequency of coronary artery abnormalities in our cohort was also within the range $(0 \%-24 \%)$ noted by these reports. Recognizing that comparative trials are not yet available in MIS-C, the use of corticosteroids, IVIG, and anakinra was associated with improvement of the inflammatory process in our patients. IVIG was used preferentially in cases with KD criteria based on its proven efficacy to prevent coronary aneurysms in KD (6). IVIG was also used in patients with myocardial dysfunction based on its efficacy for myocarditis in children (35). We elected to use anakinra for cases that were refractory to IVIG and steroids due to its efficacy in treating systemic inflammatory conditions and its overall safety profile. We have extensive experience with anakinra in the treatment of systemic JIA and it has been utilized successfully to treat severe COVID-19 in adults $(18,36,37)$. Therapeutic benefits of anakinra are also described in cases of myocarditis and KD (38, 39). Anakinra is efficacious in these conditions, despite the fact that IL-1 $\beta$ acts locally and serum IL-1 $\beta$ levels are often not detectable using standard assays. Further studies are required to determine which children warrant immunomodulatory treatment, to establish the relative efficacy of these agents, and to determine if individual agents are particularly effective against distinct manifestations of MIS-C.

A limitation of our study was the retrospective focus on hospitalized patients, without a control group of pediatric patients with COVID-19 who did not meet the case definition of MIS-C. We are only beginning to understand the pathophysiology of MIS-C, and the sensitivity and specificity of the CDC and WHO case definitions are not yet established. Not all patients who meet the current criteria may necessarily require treatment, whereas others who fall short of the case definition may still be at risk of the complications of MIS-C. Larger studies are necessary to evaluate the full spectrum of pathology associated with COVID-19 in the pediatric population and to establish risk factors for MIS-C.

In conclusion, we observed that MIS-C associated with COVID-19 is a hyperinflammatory syndrome with a broad spectrum of clinical manifestations and variable severity. MIS-C shares some features with inflammatory processes in KD and MAS, but also possesses distinct characteristics. Rapid diagnosis, multidisciplinary management, and suppression of systemic inflammation is associated with a favorable outcome in most patients.

\section{Methods}

MIS-C diagnostic criteria. Each case was reviewed by at least 4 members of the investigational team. The diagnosis of MIS- $\mathrm{C}$ was confirmed using the case definitions established by the CDC and the WHO $(14,15)$. Both the CDC and WHO case definitions are provided in Supplemental Table 1. All 28 patients fulfilled the CDC criteria and 26 patients fulfilled both case definitions, reflecting the stricter definition of fever duration and multisystem involvement in the WHO case definition. Although most MIS-C cases in our cohort were diagnosed after establishment of the CDC and WHO case definitions in mid-May, retrospective application of the definitions identified cases fulfilling the criteria for MIS-C as early as March 17, 2020 (Supplemental Figure 2C).

Comparison with KD and MAS. We compared the MIS-C cohort with patients diagnosed with KD at our center from May 2019 to February $2020(n=40)$ and a patient cohort with MAS $(n=25)$ collected between 2007 and 2019 (40). The MAS group comprised of patients with systemic juvenile idiopathic arthritis-associated (JIA-associated) MAS $(n=17)$ and infection-associated MAS $(n=8)$. The diagnosis of KD was established using the 2017 American Heart Association Guidelines (21). The diagnosis of complete KD requires the presence of 4 or all 5 principal clinical findings whereas incomplete $\mathrm{KD}$ is considered in patients with 2 or 3 criteria with suggestive laboratory and/or echocardiogram findings. The diagnosis of systemic JIA was established using the 2004 International League of Associations for Rheumatology (ILAR) Classification Criteria for JIA (41) and MAS was determined based on the 2016 ACR/EULAR Classification Criteria for MAS-complicating systemic JIA (42). While there is no formal definition for infection-associated MAS, all patients had a confirmed or likely source of infection, and met 4 or more of the 2004 Diagnostic Criteria for Hemophagocytic Lymphohistiocytosis (HLH-2004) (43). Molecular genetics were performed in 6 of these patients and mutations associated with familial HLH were not found. Triggers of infection-associated MAS included Epstein-Barr virus $(n=3)$, influenza virus $(n=2)$, Group A Streptococcus pyogenes $(n=1)$, and unknown organism causing cervical lymphadenitis $(n=1)$ or pneumonia $(n=1)$.

We separately compared the laboratory features of MIS-C with KD and with MAS (Figure 2). Therefore, laboratory parameters obtained from the different groups were not identical. For example, the MAS labs including sIL2R, ferritin, IL-18, and CXCL9 are not routinely ordered for patients with $\mathrm{KD}$.

Statistics. Median and interquartile ranges were used to express continuous variables, whereas percentages and proportions were used to express categorical variables. The differences between 2 groups were analyzed using the Mann-Whitney $U$ test. Categorical variables were compared using $\chi^{2}$ or Fisher's exact test. All tests were 2-sided, and $P$ less than 0.05 was considered significant. Statistical analyses were performed using Prism 8.0 software (GraphPad Software).

Study approval. We conducted a retrospective analysis of 28 hospitalized patients diagnosed with MIS-C at Boston Children's Hospital between March 17, 2020, and June 6, 2020. This study was approved by the Boston Children's Hospital Institutional Review Board (Boston, Massachusetts) and waiver of consent was granted for a retrospective study. Clinical manifestations and laboratory data were collected and analyzed without personal identifying information.

\section{Author contributions}

PYL, MDL, LAH and MBFS conceived and designed the study. PYL, MDL, LAH, KGF, JL, JER, MSL, CDP, JC, KJH, ALB, TMB, MHC, EC, SDDF, AD, SH, OH, JSH, MMH, EJ, EM, RWN, AAN, RPS, FD, PAN, JWN and MBFS acquired data. PYL, MDL, LAH, JL, KGF, PAN, and MBFS analyzed the data. PYL and MBFS drafted the manuscript and all authors edited the manuscript. PYL, MDL, and LAH contributed equally to data analysis. The order of co-first authorship was determined by effort in data acquisition and drafting the manuscript. 


\section{Acknowledgments}

We thank the frontline staff at Boston Children's Hospital for their dedication and excellent patient care. We thank the MAS Collaborative for helpful discussions. This work was supported by the National Institutes of Health, National Institute of Arthritis and Musculoskeletal and Skin Diseases K08-AR074562 (to PYL), K08-AR073339 (to LAH), R01-AR065538, R01AR073201, and P30-AR070253 (to PAN); the National Institute of Allergy and Infectious Diseases 5T32AI007512-34 (to JL, JR, TB, AAN, and RWN); Rheumatology Research Foundation
Investigator Awards (to PYL and LAH), and Medical Education Award (to JSH); Boston Children's Hospital Faculty Career Development Awards (to PYL and LAH); the McCance Family Foundation (to JWN); and the Samara Jan Turkel Center (to JC, RPS, MBFS).

Address correspondence to: Mary Beth F. Son and Pui Y. Lee, Boston Children's Hospital, 300 Longwood Avenue, Boston, Massachusetts 02115, USA. Phone: 617.355.6117; Email: marybeth.son@childrens. harvard.edu (MBFS); pui.lee@childrens.harvard.edu (PYL).
1. Guan WJ, et al. Clinical characteristics of coronavirus disease 2019 in China. N Engl J Med. 2020;382(18):1708-1720.

2. Huang $\mathrm{C}$, et al. Clinical features of patients infected with 2019 novel coronavirus in Wuhan, China. Lancet. 2020;395(10223):497-506.

3. Dong Y, et al. Epidemiology of COVID-19 among children in China. Pediatrics. 2020;145(6):e20200702.

4. Lu X, et al. SARS-CoV-2 infection in children. NEngl JMed. 2020;382(17):1663-1665.

5. Riphagen S, Gomez X, Gonzalez-Martinez C, Wilkinson N, Theocharis P. Hyperinflammatory shock in children during COVID-19 pandemic. Lancet . 2020;395(10237):1607-1608.

6. Verdoni L, et al. An outbreak of severe Kawasaki-like disease at the Italian epicentre of the SARS-CoV-2 epidemic: an observational cohort study. Lancet. 2020;395(10239):1771-1778.

7. Blondiaux E, et al. Cardiac MRI of children with multisystem inflammatory syndrome (MIS-C) associated with COVID-19: case series [published online June 9, 2020]. Radiology. https:// doi.org/10.1148/radiol.2020202288.

8. Dufort EM, et al. Multisystem inflammatory syndrome in children in New York State. $N$ Engl J Med. 2020;383(4):347-358.

9. Feldstein LR, et al. Multisystem inflammatory syndrome in U.S. children and adolescents. N Engl JMed. 2020;383(4):334-346.

10. Toubiana J, et al. Kawasaki-like multisystem inflammatory syndrome in children during the COVID-19 pandemic in Paris, France: prospective observational study. BMJ. 2020;369:m2094.

11. Shekerdemian LS, et al. Characteristics and outcomes of children with coronavirus disease 2019 (COVID-19) infection admitted to US and Canadian pediatric intensive care units [published online May 11, 2020]. JAMA Pediatr. https://doi. org/10.1001/jamapediatrics.2020.1948.

12. Belhadjer Z, et al. Acute heart failure in multisystem inflammatory syndrome in children (MIS-C) in the context of global SARS-CoV-2 pandemic [published online May 17, 2020]. Circulation. https://doi. org/10.1161/circulationaha.120.048360.

13. Guidance: Paediatric multisystem inflammatory syndrome temporally associated with COVID19 (PIMS). Royal College of Paediatrics and Child Health. https://www.rcpch.ac.uk/resources/guidance-paediatric-multisystem-inflammatory-syndrome-temporally-associated-covid-19-pims. Published May 1, 2020. Accessed October 1, 2020.

14. CDC. Multisystem inflammatory syndrome in children (MIS-C) associated with coronavirus disease 2019 (COVID-19). https://www.cdc. gov/coronavirus/2019-ncov/daily-life-coping/ children/mis-c.html. Updated May 20, 2020. Accessed October 1, 2020.

15. WHO. Multisystem inflammatory syndrome in children and adolescents with COVID-19. https://www.who.int/publications/i/item/ multisystem-inflammatory-syndrome-in-children-and-adolescents-with-covid-19. Updated May 15, 2020. Accessed August 25, 2020.

16. Chen G, et al. Clinical and immunological features of severe and moderate coronavirus disease 2019. J Clin Invest. 2020;130(5):2620-2629.

17. Henderson LA, et al. On the alert for cytokine storm: immunopathology in COVID-19. Arthritis Rheumatol. 2020;72(7):1059-1063..

18. Pontali E, et al. Safety and efficacy of early highdose IV anakinra in severe COVID-19 lung disease. J Allergy Clin Immunol. 2020;146(1):213-215

19. Toniati $P$, et al. Tocilizumab for the treatment of severe COVID-19 pneumonia with hyperinflammatory syndrome and acute respiratory failure: A single center study of 100 patients in Brescia, Italy. Autoimmun Rev. 2020;19(7):102568.

20. United States Census Bureau. QuickFacts - Boston and Massachusetts. https://www.census.gov/ quickfacts/bostoncitymassachusetts. Accessed June 1, 2020.

21. McCrindle BW, et al. Diagnosis, treatment, and long-term management of Kawasaki disease: a scientific statement for health professionals from the American Heart Association. Circulation. 2017;135(17):e927-e999.

22. Strouse JJ, Takemoto CM, Keefer JR, Kato GJ, Casella JF. Corticosteroids and increased risk of readmission after acute chest syndrome in children with sickle cell disease. Pediatr Blood Cancer. 2008;50(5):1006-1012.

23. Levi M, Thachil J, Iba T, Levy JH. Coagulation abnormalities and thrombosis in patients with COVID-19. Lancet Haematol. 2020;7(6): e438-e440.

24. Cheung EW, et al. Multisystem inflammatory syndrome related to COVID-19 in previously healthy children and adolescents in New York City. JAMA. 2020;324(3):294-296.

25. Whittaker E, et al. Clinical characteristics of 58 children with a pediatric inflammatory multisystem syndrome temporally associated with SARS-CoV-2 [June 8, 2020]. JAMA. https://doi. org/10.1001/jama.2020.10369.

26. Webb Hooper M, Nápoles AM, Pérez-Stable EJ. COVID-19 and racial/ethnic disparities [published online May 11, 2020]. JAMA. https://doi. org/10.1001/jama.2020.8598.
27. Wadhera RK, et al. Variation in COVID-19 hospitalizations and deaths across New York City boroughs. JAMA. 2020;323(21):2192-2195.

28. CDC COVID-19 Response Team. Preliminary estimates of the prevalence of selected underlying health conditions among patients with coronavirus disease 2019 - United States, February 12-March 28, 2020. MMWR Morb Mortal Wkly Rep. 2020;69(13):382-386.

29. Herold T, et al. Elevated levels of IL-6 and CRP predict the need for mechanical ventilation in COVID-19. JAllergy Clin Immunol. 2020;146(1):128-136.e4.

30. Li S, et al. Clinical and pathological investigation of patients with severe COVID-19. JCI Insight. 2020;5(12):e138070.

31. Put K, et al. Cytokines in systemic juvenile idiopathic arthritis and haemophagocytic lymphohistiocytosis: tipping the balance between interleukin-18 and interferon- $\gamma$. Rheumatology (Oxford). 2015;54(8):1507-1517.

32. Ye Q, Shao WX, Shang SQ, Zhang T, Hu J, Zhang CC. A comprehensive assessment of the value of laboratory indices in diagnosing Kawasaki disease. Arthritis Rheumatol. 2015;67(7):1943-1950.

33. Chiotos $\mathrm{K}$, et al. Multisystem inflammatory syndrome in children during the coronavirus 2019 pandemic: a case series. J Pediatric Infect Dis Soc. 2020;9(3):393-398.

34. Grimaud M, et al. Acute myocarditis and multisystem inflammatory emerging disease following SARS-CoV-2 infection in critically ill children. Ann Intensive Care. 2020;10(1):69.

35. Drucker NA, et al. Gamma-globulin treatment of acute myocarditis in the pediatric population. Circulation. 1994;89(1):252-257.

36. Aouba A, et al. Targeting the inflammatory cascade with anakinra in moderate to severe COVID-19 pneumonia: case series [published online May 6, 2020]. Ann Rheum Dis. https://doi. org/10.1136/annrheumdis-2020-217706.

37. Cavalli G, et al. Interleukin-1 blockade with highdose anakinra in patients with COVID-19, acute respiratory distress syndrome, and hyperinflammation: a retrospective cohort study. Lancet Rheumatol. 2020;2(6):e325-e331.

38. Cavalli G, Pappalardo F, Mangieri A, Dinarello CA, Dagna L, Tresoldi M. Treating life-threatening myocarditis by blocking interleukin-1. Crit Care Med. 2016;44(8):e751-754.

39. Cohen S, Tacke CE, Straver B, Meijer N, Kuipers IM, Kuijpers TW. A child with severe relapsing Kawasaki disease rescued by IL-1 receptor blockade and extracorporeal membrane oxygenation. 
Ann Rheum Dis. 2012;71(12):2059-2061.

40. Lee PY, et al. Adenosine deaminase 2 as a biomarker of macrophage activation syndrome in systemic juvenile idiopathic arthritis. Ann Rheum Dis. 2020;79(2):225-231.

41. Petty RE, et al. International League of Associations for Rheumatology classification of juvenile idiopathic arthritis: second revision, Edmonton, 2001. J Rheumatol. 2004;31(2):390-392.

42. Ravelli A, et al. 2016 classification criteria for macrophage activation syndrome complicating systemic juvenile idiopathic arthritis: A European League Against Rheumatism/American College of Rheumatology/Paediatric Rheu- matology International Trials Organisation Collaborative Initiative. Arthritis Rheumatol. 2016;68(3):566-576.

43. Henter JI, et al. HLH-2004: diagnostic and therapeutic guidelines for hemophagocytic lymphohistiocytosis. Pediatr Blood Cancer. 2007;48(2):124-131. 